Acta Crystallographica Section E

Structure Reports

Online

ISSN 1600-5368

David J. Watkin, ${ }^{a} *$ W. D. S. Motherwell, ${ }^{\mathrm{b}}$ Richard I. Cooper, Stefan Pantos ${ }^{\mathrm{a}}$ and Oliver I. Steadman ${ }^{\mathrm{a}}$

${ }^{a}$ Chemical Crystallography, Chemistry Research Laboratory, University of Oxford, Mansfield Road, Oxford OX1 3TA, England, and ${ }^{\mathbf{b}} \mathrm{Cam}$ Cambridge Crystallographic Data Centre, 12 Union Road, Cambridge CB2 1EZ, England

Correspondence e-mail:

david.watkin@chem.ox.ac.uk

\section{Key indicators}

Single-crystal X-ray study

$T=120 \mathrm{~K}$

Mean $\sigma(\mathrm{C}-\mathrm{C})=0.002 \AA$

$R$ factor $=0.024$

$w R$ factor $=0.062$

Data-to-parameter ratio $=7.7$

For details of how these key indicators were automatically derived from the article, see http://journals.iucr.org/e.

\title{
$N$-(2-Methyl-3,6-dinitrophenyl)acetamide
}

The structure of the title compound, $\mathrm{C}_{9} \mathrm{H}_{9} \mathrm{~N}_{3} \mathrm{O}_{5}$, was determined as one of a group of five related compounds in order to assess its suitability as a test material for the 2004 Cambridge Crystallographic Data Centre 'Blind Structure Prediction Test'. The structure consists of hydrogen-bonded ribbons of molecules stacked along the $a$ axis with the benzene rings parallel by unit-cell translations.

\section{Comment}

The structure of the title material, (I), was determined as part of the preparations for the 2004 Cambridge Crystallographic Data Centre 'Blind Structure Prediction Tests' (Watkin et al., 2004), though (I) was not used in the test. The material was from a collection of nitrotoluene derivatives synthesized by Wilhelm Koerner about a century ago and retrieved from a depository at the University of Milan.<smiles>CC(=O)Nc1c([N+](=O)[O-])ccc([N+](=O)[O-])c1C</smiles>

(I)

The sample consisted of large, striated, pale-cream laths. Attempts were made to obtain a roughly isometric sample, but the specimens inevitably splintered freely if any attempt was

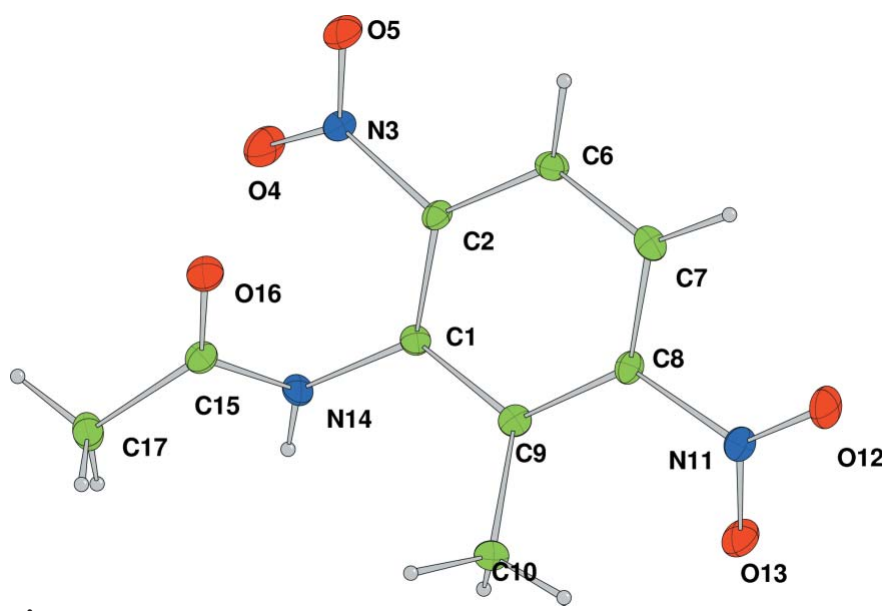

Figure 1

The title compound, with atomic displacement parameters drawn at the $50 \%$ probability level and the $\mathrm{H}$ atoms with arbitary radii.
Received 17 October 2005 Accepted 24 October 2005 Online 27 October 2005
(C) 2005 International Union of Crystallography Printed in Great Britain - all rights reserved 
made to cut them into shorter lengths. One was selected on the basis of its sharp diffraction pattern and relative thickness. Changes in illuminated volume were kept to a minimum by the data collection strategy, and were taken into account (Görbitz, 1999) by the multi-scan inter-frame scaling (DENZO/SCALEPACK; Otwinowski \& Minor, 1997).

The two nitro groups are twisted by almost the same angle from the plane of the benzene ring $[\mathrm{C} 1-\mathrm{C} 2-\mathrm{N} 3-\mathrm{O} 5=$ $143.3(3)^{\circ}$ and $\left.\mathrm{C} 9-\mathrm{C} 8-\mathrm{C} 11-\mathrm{O} 12=-147.9(3)^{\circ}\right]$. The almost planar acetamide group is rotated out of the ring plane [C9$\mathrm{C} 1-\mathrm{N} 14-\mathrm{C} 15=129.3(3)^{\circ}$ ] (Fig. 1).

The structure consists of ribbons of molecules stacked with the benzene rings parallel by unit-cell translations along the $a$ axis, giving an interplanar separation of 3.618 (3) § (Fig. 2). Molecules in these ribbons are linked together by hydrogen bonds (Fig. 3 and Table 1). Other intermolecular contacts are unexceptional.

\section{Experimental}

The material was from a collection of nitrotoluene derivatives synthesized by Wilhelm Koerner about a century ago and retrieved from a depository at the University of Milan (Demartin et al., 2004). Details of the preparation and crystallization are unknown.

\section{Crystal data}

$\mathrm{C}_{9} \mathrm{H}_{9} \mathrm{~N}_{3} \mathrm{O}_{5}$

$M_{r}=239.19$

Monoclinic, $P 2_{1}$

$a=4.9309$ (2) А

$b=11.7571$ (4) $\AA$

$c=8.7944(3) \AA$

$\beta=99.8608(14)^{\circ}$

$V=502.31(3) \AA^{3}$

$Z=2$

Data collection

Nonius KappaCCD diffractometer $\omega$ scans

Absorption correction: multi-scan

(DENZO/SCALEPACK;

Otwinowski \& Minor, 1997)

$T_{\min }=0.80, T_{\max }=0.99$

3452 measured reflections

\section{Refinement}

Refinement on $F^{2}$

$R\left[F^{2}>2 \sigma\left(F^{2}\right)\right]=0.024$

$w R\left(F^{2}\right)=0.062$

$S=1.09$

1188 reflections

154 parameters

Table 1

Hydrogen-bond geometry $\left(\AA,^{\circ}\right)$.

\begin{tabular}{lllll}
\hline$D-\mathrm{H} \cdots A$ & $D-\mathrm{H}$ & $\mathrm{H} \cdots A$ & $D \cdots A$ & $D-\mathrm{H} \cdots A$ \\
\hline $\mathrm{N} 14-\mathrm{H} 7 \cdots \mathrm{O}^{\mathrm{i}}{ }^{\mathrm{i}}$ & 0.84 & 2.13 & $2.963(2)$ & 168 \\
\hline
\end{tabular}

Symmetry code: (i) $x-1, y, z$.

In the absence of significant anomalous scattering, Friedel pairs were merged. The $\mathrm{H}$ atoms were all located in a difference map, but

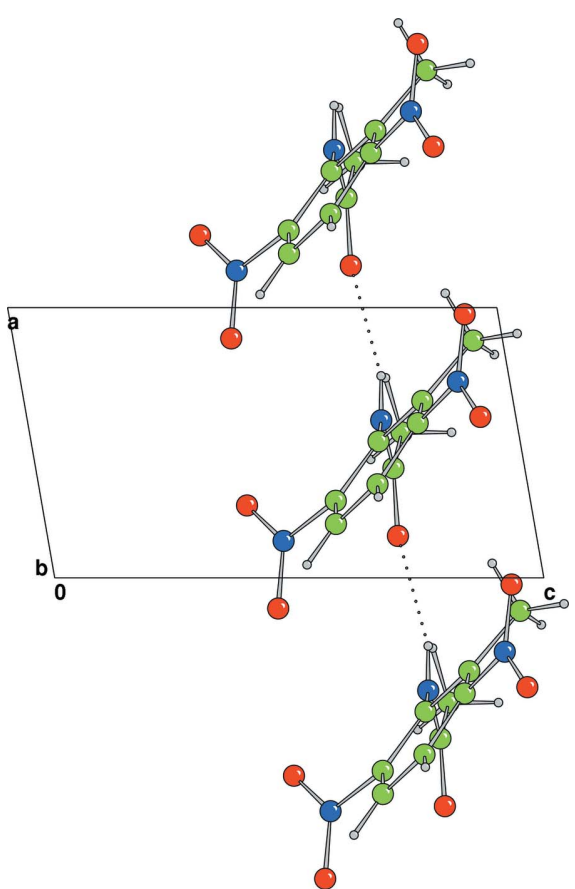

Figure 2

Projection along the $b$ axis, showing the hydrogen bonding (dotted lines) and the aromatic ring stacking.

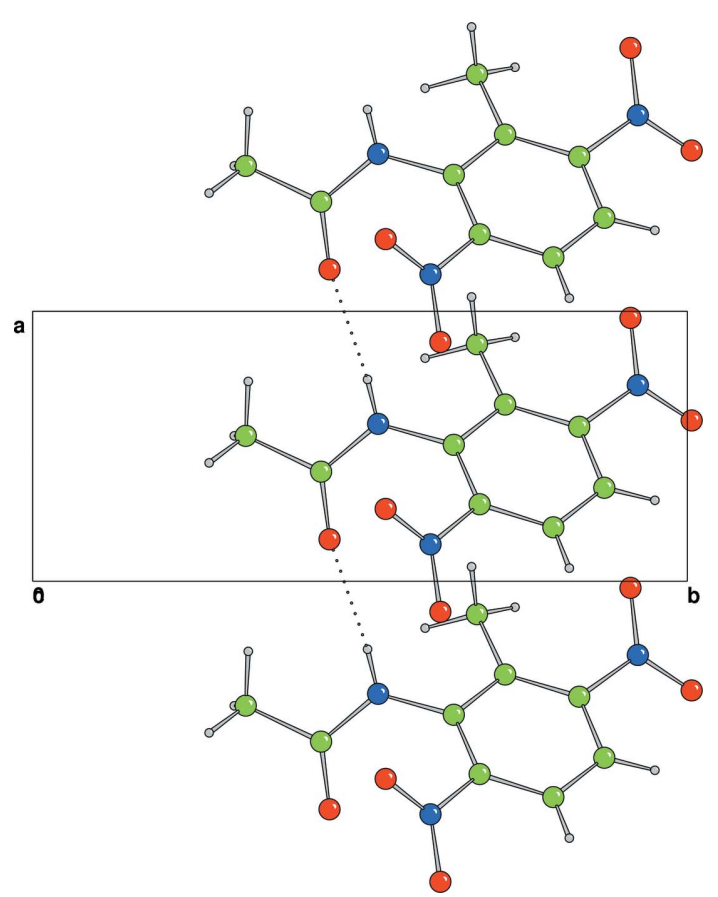

Figure 3

Projection along the $a$ axis, showing the hydrogen-bonded (dotted lines) chain, with the benzene rings all on the same side of the $c$ axis.

those attached to $\mathrm{C}$ atoms were repositioned geometrically. The $\mathrm{H}$ atoms were initially refined with soft restraints on the bond lengths and angles to regularize their geometry $(\mathrm{C}-\mathrm{H}$ in the target range 0.93-98 $\AA$ and $\mathrm{N}-\mathrm{H} 0.86 \AA$ ) and isotropic displacement parameters [ $U_{\text {iso }}(\mathrm{H})$ in the range $1.2-1.5$ times $U_{\text {eq }}$ of the parent atom], after which they were refined with riding constraints. 
Data collection: COLLECT (Nonius, 2001); cell refinement: DENZO/SCALEPACK (Otwinowski \& Minor, 1997); data reduction: DENZO/SCALEPACK; program(s) used to solve structure: SIR92 (Altomare et al., 1994); program(s) used to refine structure: CRYSTALS (Betteridge et al., 2003); molecular graphics: CAMERON (Watkin et al., 1996); software used to prepare material for publication: CRYSTALS.

We thank Professor Angelo Gavezzotti for obtaining the samples, Professor Lucio Merlini, Director of the Dipartmento di Scienze Molecolari Agroalimentari of the University of Milan, for generously donating them, and Professor Anna Arnoldi for help in their retrieval.

\section{References}

Altomare, A., Cascarano, G., Giacovazzo, G., Guagliardi, A., Burla, M. C., Polidori, G. \& Camalli, M. (1994). J. Appl. Cryst. 27, 435.

Betteridge, P. W., Carruthers, J. R., Cooper, R. I., Prout, K. \& Watkin, D. J. (2003). J. Appl. Cryst. 36, 1487.

Demartin, F., Filippini, G., Gavezzotti, A. \& Rizzato, S. (2004). Acta Cryst. B60, 609-620.

Görbitz, C. H. (1999). Acta Cryst. B55, 1090-1098.

Nonius (2001). COLLECT. Nonius BV, Delft, The Netherlands.

Otwinowski, Z. \& Minor, W. (1997). Methods in Enzymology, Vol. 276, Macromolecular Crystallography, Part A, edited by C. W. Carter Jr \& R. M. Sweet, pp. 307-326. New York: Academic Press.

Watkin, D. J., Motherwell, W. D. S., Cooper, R. I. \& Pantos, S. (2004). Acta Cryst. E60, o2295-02297.

Watkin, D. J., Prout, C. K. \& Pearce, L. J. (1996). CAMERON. Chemical Crystallography Laboratory, Oxford, England. 


\section{supporting information}

Acta Cryst. (2005). E61, o3888-o3890 [https://doi.org/10.1107/S1600536805034355]

\section{N-(2-Methyl-3,6-dinitrophenyl)acetamide}

David J. Watkin, W. D. S. Motherwell, Richard I. Cooper, Stefan Pantos and Oliver I. Steadman

N-(2-Methyl-3,6-dinitrophenyl)acetamide

Crystal data

$\mathrm{C}_{9} \mathrm{H}_{9} \mathrm{~N}_{3} \mathrm{O}_{5}$

$M_{r}=239.19$

Monoclinic, $P 2_{1}$ $a=4.9309(2) \AA$

$b=11.7571(4) \AA$

$c=8.7944(3) \AA$

$\beta=99.8608(14)^{\circ}$

$V=502.31(3) \AA^{3}$

$Z=2$

$F(000)=248$

$D_{\mathrm{x}}=1.581 \mathrm{Mg} \mathrm{m}^{-3}$

Mo $K \alpha$ radiation, $\lambda=0.71073 \AA$

Cell parameters from 939 reflections

$\theta=5-27^{\circ}$

$\mu=0.13 \mathrm{~mm}^{-1}$

$T=120 \mathrm{~K}$

Lath, pale-yellow

$0.76 \times 0.20 \times 0.10 \mathrm{~mm}$

\section{Data collection}

Nonius KappaCCD

3452 measured reflections diffractometer

Graphite monochromator

$\omega$ scans

Absorption correction: multi-scan

(DENZO/SCALEPACK; Otwinowski \& Minor, 1997)

$T_{\min }=0.80, T_{\max }=0.99$

1188 independent reflections

1188 reflections with $I>-10 \sigma(I)$

$R_{\text {int }}=0.021$

$\theta_{\max }=27.5^{\circ}, \theta_{\min }=5.2^{\circ}$

$h=-6 \rightarrow 6$

$k=-15 \rightarrow 12$

$l=-11 \rightarrow 11$

Refinement

Refinement on $F^{2}$

Least-squares matrix: full

$R\left[F^{2}>2 \sigma\left(F^{2}\right)\right]=0.024$

$w R\left(F^{2}\right)=0.062$

$S=1.09$

1188 reflections

154 parameters

1 restraint

Primary atom site location: structure-invariant direct methods

Hydrogen site location: inferred from neighbouring sites

$\mathrm{H}$-atom parameters constrained

$w=1 /\left[\sigma^{2}\left(F^{2}\right)+(0.02 P)^{2}+0.13 P\right]$

where $P=\left[\max \left(F_{\mathrm{o}}^{2}, 0\right)+2 F_{\mathrm{c}}^{2}\right] / 3$

$(\Delta / \sigma)_{\max }=0.000205$

$\Delta \rho_{\max }=0.21$ e $\AA^{-3}$

$\Delta \rho_{\min }=-0.17$ e $\AA^{-3}$

Fractional atomic coordinates and isotropic or equivalent isotropic displacement parameters $\left(\AA^{2}\right)$

\begin{tabular}{lllll}
\hline & $x$ & $y$ & $z$ & $U_{\text {iso }} * / U_{\text {eq }}$ \\
\hline C1 & $0.4948(3)$ & $0.14319(14)$ & $0.28928(18)$ & 0.0111 \\
C2 & $0.7148(3)$ & $0.18283(14)$ & $0.39815(18)$ & 0.0114 \\
N3 & $0.8638(3)$ & $0.10778(13)$ & $0.51881(15)$ & 0.0140 \\
O4 & $0.7326(3)$ & $0.03921(12)$ & $0.58113(14)$ & 0.0219
\end{tabular}




$\begin{array}{lllll}\text { O5 } & 1.1140(2) & 0.12260(12) & 0.55405(15) & 0.0219 \\ \text { C6 } & 0.8003(3) & 0.29526(15) & 0.40571(18) & 0.0134 \\ \text { C7 } & 0.6540(3) & 0.37335(15) & 0.30692(19) & 0.0139 \\ \text { C8 } & 0.4280(3) & 0.33503(15) & 0.20287(18) & 0.0116 \\ \text { C9 } & 0.3458(3) & 0.22171(15) & 0.18546(18) & 0.0118 \\ \text { C10 } & 0.1222(3) & 0.17863(15) & 0.06026(19) & 0.0159 \\ \text { N11 } & 0.2738(3) & 0.42462(13) & 0.10628(16) & 0.0138 \\ \text { O12 } & 0.4046(3) & 0.50665(11) & 0.07238(15) & 0.0211 \\ \text { O13 } & 0.0249(2) & 0.41328(11) & 0.06852(15) & 0.0196 \\ \text { N14 } & 0.4172(3) & 0.02758(12) & 0.27649(17) & 0.0130 \\ \text { C15 } & 0.5946(3) & -0.05962(14) & 0.26833(18) & 0.0129 \\ \text { O16 } & 0.8450(2) & -0.04647(11) & 0.28353(14) & 0.0166 \\ \text { C17 } & 0.4615(3) & -0.17467(14) & 0.2390(2) & 0.0169 \\ \text { H61 } & 0.9522 & 0.3189 & 0.4777 & 0.0171^{*} \\ \text { H71 } & 0.7032 & 0.4511 & 0.3102 & 0.0169^{*} \\ \text { H73 } & 0.2672 & -0.1711 & 0.2518 & 0.0263^{*} \\ \text { H72 } & 0.4683 & -0.1941 & 0.1353 & 0.0281^{*} \\ \text { H4 } & 0.5585 & -0.2301 & 0.3078 & 0.0284^{*} \\ \text { H7 } & 0.2481 & 0.0118 & 0.2655 & 0.0178^{*} \\ \text { H1 } & 0.1742 & 0.1034 & 0.0229 & 0.0241^{*} \\ \text { H2 } & -0.0511 & 0.1712 & 0.1004 & 0.0240^{*} \\ \text { H3 } & 0.0949 & 0.2334 & -0.0280 & 0.0238^{*}\end{array}$

Atomic displacement parameters $\left(\AA^{2}\right)$

\begin{tabular}{lllllll}
\hline & $U^{11}$ & $U^{22}$ & $U^{33}$ & $U^{12}$ & $U^{13}$ & $U^{23}$ \\
\hline C1 & $0.0084(6)$ & $0.0117(8)$ & $0.0136(7)$ & $-0.0006(6)$ & $0.0031(5)$ & $-0.0001(6)$ \\
C2 & $0.0089(7)$ & $0.0124(8)$ & $0.0129(7)$ & $0.0016(6)$ & $0.0016(5)$ & $0.0019(6)$ \\
N3 & $0.0133(6)$ & $0.0144(7)$ & $0.0136(6)$ & $0.0012(6)$ & $-0.0001(5)$ & $0.0007(6)$ \\
O4 & $0.0207(6)$ & $0.0245(7)$ & $0.0203(6)$ & $-0.0025(6)$ & $0.0027(5)$ & $0.0099(6)$ \\
O5 & $0.0124(5)$ & $0.0206(7)$ & $0.0300(7)$ & $0.0002(5)$ & $-0.0045(5)$ & $0.0049(6)$ \\
C6 & $0.0111(7)$ & $0.0141(8)$ & $0.0144(7)$ & $-0.0009(6)$ & $0.0004(5)$ & $-0.0018(7)$ \\
C7 & $0.0141(7)$ & $0.0122(7)$ & $0.0159(8)$ & $-0.0014(6)$ & $0.0040(6)$ & $-0.0024(6)$ \\
C8 & $0.0114(7)$ & $0.0114(7)$ & $0.0127(7)$ & $0.0016(6)$ & $0.0035(6)$ & $0.0016(6)$ \\
C9 & $0.0093(6)$ & $0.0142(8)$ & $0.0127(7)$ & $0.0003(6)$ & $0.0040(6)$ & $0.0002(6)$ \\
C10 & $0.0134(7)$ & $0.0150(8)$ & $0.0178(8)$ & $-0.0005(6)$ & $-0.0014(6)$ & $-0.0006(7)$ \\
N11 & $0.0140(6)$ & $0.0135(7)$ & $0.0141(6)$ & $0.0017(6)$ & $0.0032(5)$ & $0.0011(6)$ \\
O12 & $0.0209(6)$ & $0.0149(6)$ & $0.0270(7)$ & $-0.0034(5)$ & $0.0026(5)$ & $0.0083(5)$ \\
O13 & $0.0124(5)$ & $0.0192(7)$ & $0.0262(6)$ & $0.0027(5)$ & $0.0009(5)$ & $0.0042(6)$ \\
N14 & $0.0080(5)$ & $0.0115(7)$ & $0.0194(7)$ & $-0.0012(5)$ & $0.0020(5)$ & $-0.0001(6)$ \\
C15 & $0.0132(7)$ & $0.0138(8)$ & $0.0120(7)$ & $0.0008(6)$ & $0.0024(5)$ & $0.0014(6)$ \\
O16 & $0.0099(5)$ & $0.0176(6)$ & $0.0225(6)$ & $0.0010(5)$ & $0.0027(4)$ & $0.0009(5)$ \\
C17 & $0.0156(7)$ & $0.0121(8)$ & $0.0230(8)$ & $-0.0002(7)$ & $0.0032(6)$ & $0.0003(7)$ \\
& & & & & &
\end{tabular}

Geometric parameters $(\AA, \stackrel{\circ}{)})$

\begin{tabular}{llll}
\hline $\mathrm{C} 1-\mathrm{C} 2$ & $1.398(2)$ & $\mathrm{C} 9-\mathrm{C} 10$ & $1.506(2)$ \\
$\mathrm{C} 1-\mathrm{C} 9$ & $1.413(2)$ & $\mathrm{C} 10-\mathrm{H} 1$ & 0.992
\end{tabular}




\begin{tabular}{|c|c|c|c|}
\hline $\mathrm{C} 1-\mathrm{N} 14$ & $1.411(2)$ & $\mathrm{C} 10-\mathrm{H} 2$ & 0.982 \\
\hline $\mathrm{C} 2-\mathrm{N} 3$ & $1.476(2)$ & $\mathrm{C} 10-\mathrm{H} 3$ & 1.000 \\
\hline $\mathrm{C} 2-\mathrm{C} 6$ & $1.386(2)$ & $\mathrm{N} 11-\mathrm{O} 12$ & $1.2250(19)$ \\
\hline $\mathrm{N} 3-\mathrm{O} 4$ & $1.221(2)$ & $\mathrm{N} 11-\mathrm{O} 13$ & $1.2228(17)$ \\
\hline $\mathrm{N} 3-\mathrm{O} 5$ & $1.2318(17)$ & N14-C15 & $1.358(2)$ \\
\hline $\mathrm{C} 6-\mathrm{C} 7$ & $1.380(2)$ & $\mathrm{N} 14-\mathrm{H} 7$ & 0.843 \\
\hline $\mathrm{C} 6-\mathrm{H} 61$ & 0.936 & $\mathrm{C} 15-\mathrm{O} 16$ & $1.2287(18)$ \\
\hline $\mathrm{C} 7-\mathrm{C} 8$ & $1.390(2)$ & $\mathrm{C} 15-\mathrm{C} 17$ & $1.506(2)$ \\
\hline $\mathrm{C} 7-\mathrm{H} 71$ & 0.945 & $\mathrm{C} 17-\mathrm{H} 73$ & 0.984 \\
\hline $\mathrm{C} 8-\mathrm{C} 9$ & $1.393(2)$ & $\mathrm{C} 17-\mathrm{H} 72$ & 0.947 \\
\hline $\mathrm{C} 8-\mathrm{N} 11$ & $1.479(2)$ & $\mathrm{C} 17-\mathrm{H} 4$ & 0.959 \\
\hline $\mathrm{C} 2-\mathrm{C} 1-\mathrm{C} 9$ & $118.77(15)$ & $\mathrm{C} 9-\mathrm{C} 10-\mathrm{H} 1$ & 110.1 \\
\hline $\mathrm{C} 2-\mathrm{C} 1-\mathrm{N} 14$ & $122.93(15)$ & $\mathrm{C} 9-\mathrm{C} 10-\mathrm{H} 2$ & 109.9 \\
\hline $\mathrm{C} 9-\mathrm{C} 1-\mathrm{N} 14$ & 118.29 (14) & $\mathrm{H} 1-\mathrm{C} 10-\mathrm{H} 2$ & 109.2 \\
\hline $\mathrm{C} 1-\mathrm{C} 2-\mathrm{N} 3$ & $121.71(15)$ & $\mathrm{C} 9-\mathrm{C} 10-\mathrm{H} 3$ & 109.6 \\
\hline $\mathrm{C} 1-\mathrm{C} 2-\mathrm{C} 6$ & $123.02(15)$ & $\mathrm{H} 1-\mathrm{C} 10-\mathrm{H} 3$ & 109.0 \\
\hline $\mathrm{N} 3-\mathrm{C} 2-\mathrm{C} 6$ & $115.20(14)$ & $\mathrm{H} 2-\mathrm{C} 10-\mathrm{H} 3$ & 109.1 \\
\hline $\mathrm{C} 2-\mathrm{N} 3-\mathrm{O} 4$ & $118.72(13)$ & $\mathrm{C} 8-\mathrm{N} 11-\mathrm{O} 12$ & $117.40(13)$ \\
\hline $\mathrm{C} 2-\mathrm{N} 3-\mathrm{O} 5$ & $116.77(14)$ & $\mathrm{C} 8-\mathrm{N} 11-\mathrm{O} 13$ & $118.15(14)$ \\
\hline $\mathrm{O} 4-\mathrm{N} 3-\mathrm{O} 5$ & $124.44(14)$ & $\mathrm{O} 12-\mathrm{N} 11-\mathrm{O} 13$ & $124.44(15)$ \\
\hline $\mathrm{C} 2-\mathrm{C} 6-\mathrm{C} 7$ & $118.84(15)$ & $\mathrm{C} 1-\mathrm{N} 14-\mathrm{C} 15$ & $124.26(14)$ \\
\hline $\mathrm{C} 2-\mathrm{C} 6-\mathrm{H} 61$ & 121.1 & $\mathrm{C} 1-\mathrm{N} 14-\mathrm{H} 7$ & 118.0 \\
\hline $\mathrm{C} 7-\mathrm{C} 6-\mathrm{H} 61$ & 120.0 & $\mathrm{C} 15-\mathrm{N} 14-\mathrm{H} 7$ & 117.5 \\
\hline $\mathrm{C} 6-\mathrm{C} 7-\mathrm{C} 8$ & $118.21(16)$ & $\mathrm{N} 14-\mathrm{C} 15-\mathrm{O} 16$ & $122.96(16)$ \\
\hline $\mathrm{C} 6-\mathrm{C} 7-\mathrm{H} 71$ & 121.4 & $\mathrm{~N} 14-\mathrm{C} 15-\mathrm{C} 17$ & $114.91(13)$ \\
\hline $\mathrm{C} 8-\mathrm{C} 7-\mathrm{H} 71$ & 120.4 & $\mathrm{O} 16-\mathrm{C} 15-\mathrm{C} 17$ & $122.14(15)$ \\
\hline $\mathrm{C} 7-\mathrm{C} 8-\mathrm{C} 9$ & $124.57(15)$ & $\mathrm{C} 15-\mathrm{C} 17-\mathrm{H} 73$ & 110.3 \\
\hline $\mathrm{C} 7-\mathrm{C} 8-\mathrm{N} 11$ & $114.99(15)$ & $\mathrm{C} 15-\mathrm{C} 17-\mathrm{H} 72$ & 107.2 \\
\hline $\mathrm{C} 9-\mathrm{C} 8-\mathrm{N} 11$ & $120.43(13)$ & $\mathrm{H} 73-\mathrm{C} 17-\mathrm{H} 72$ & 108.5 \\
\hline $\mathrm{C} 1-\mathrm{C} 9-\mathrm{C} 8$ & $116.39(14)$ & $\mathrm{C} 15-\mathrm{C} 17-\mathrm{H} 4$ & 110.2 \\
\hline $\mathrm{C} 1-\mathrm{C} 9-\mathrm{C} 10$ & $119.16(15)$ & $\mathrm{H} 73-\mathrm{C} 17-\mathrm{H} 4$ & 110.3 \\
\hline $\mathrm{C} 8-\mathrm{C} 9-\mathrm{C} 10$ & $124.37(15)$ & $\mathrm{H} 72-\mathrm{C} 17-\mathrm{H} 4$ & 110.4 \\
\hline
\end{tabular}

Hydrogen-bond geometry $\left(\AA,{ }^{\circ}\right)$

\begin{tabular}{lllll}
\hline$D-\mathrm{H} \cdots A$ & $D-\mathrm{H}$ & $\mathrm{H} \cdots A$ & $D \cdots A$ & $D-\mathrm{H} \cdots A$ \\
\hline $\mathrm{N} 14-\mathrm{H} 7 \cdots \mathrm{O} 16^{\mathrm{i}}$ & 0.84 & 2.13 & $2.963(2)$ & 168 \\
\hline
\end{tabular}

Symmetry code: (i) $x-1, y, z$. 